\title{
Amplification of c-myc Oncogene by Chromogenic and Fluorescence In Situ Hybridization in Archival Breast Cancer Tissue Array Samples
}

\author{
Jaana K. Rummukainen, Tiina Salminen, Johan Lundin, Heikki Joensuu, and \\ Jorma J. Isola \\ Institute of Medical Technology (JKR, JJI), University of Tampere and Tampere University Hospital, Tampere; and \\ Department of Oncology (TS, JL, HJ), Helsinki University Central Hospital, Helsinki, Finland
}

SUMMARY: Fluorescence in situ hybridization (FISH) is currently considered to be the most specific and sensitive method for detection of oncogene amplifications in human tumor samples. However, FISH requires fluorescence microscopy, which is tedious and does not allow histopathologic evaluation of the cells and tissues examined. Here we compared FISH with the newly developed chromogenic in situ hybridization (CISH), which uses peroxidase enzyme for probe detection instead of fluorescent dyes. $\mathrm{CISH}$ was found to be highly concordant with FISH in a tissue array series of 177 archival breast cancer samples. This was true both when comparing $\mathrm{CISH}$ with single-color and two-color $\mathrm{FISH}$, the latter including the chromosome 8 centromere probe as reference (the kappa coefficients were 0.67 and 0.76 , respectively). Clinicopathologic correlations of c-myc amplification as detected by FISH and $\mathrm{CISH}$ were generally the same. By both methods, c-myc amplification was significantly associated with high histologic grade, negative progesterone receptor status, DNA aneuploidy, and high S-phase fraction. c-myc amplification was strongly associated with poor distant metastasis-free survival when amplification was detected by $\mathrm{CISH}(p=0.0013)$, but this association was weaker when FISH was used ( $p=0.16$ for two-color FISH and $p=0.065$ for single-color FISH). These data suggest that $\mathrm{CISH}$ is at least as sensitive and specific as FISH in the detection of oncogene amplification in human tumor samples. The possibility for concomitant tissue architecture evaluation using an ordinary transmitted light microscope may favor the use of $\mathrm{CISH}$ over FISH in oncogene amplification detection in large tumor series, and tissue arrays and, ultimately, in routine clinical diagnostics. (Lab Invest 2001, 81:1545-1551).

$$
T^{\text {has }}
$$
he c-myc oncogene is amplified in breast cancer as well as in several other malignancies in a large number of studies (Escot et al, 1986; Nesbit et al, 1999; Visscher et al, 1997). However, the proportion of tumors with c-myc amplification ranges widely, from $1 \%$ to $94 \%$ in different studies (Deming et al, 2000). The source of such variation may lie at least in part in the inconsistencies of the assay methods. Most studies have been performed using Southern blotting, which is known to suffer from normal cell contamination of the tumor sample (Nesbit et al, 1999). Immunohistochemical staining of the c-myc protein overexpression has never become as widely used as in the case of the HER-2/neu oncogene.

Fluorescence in situ hybridization (FISH) is currently considered the most accurate method for detection of oncogene amplification in human tumors (Persons et al, 1997; Press et al, 1997; Tubbs et al, 2001), but FISH has been applied to c-myc amplification detection in only four studies of breast cancer. In these studies the proportion of tumors with c-myc amplification was

Received July 20, 2001

This study was financially supported by The Finnish Cancer Society and the Tampere University Hospital Research Foundation.

Address reprint requests to: Dr. Jorma Isola, Institute of Medical Technology, FIN-33101 University of Tampere, Finland.E-mail: jorma.isola@uta.fi
12\% (Schraml et al, 1999), 14.6\% (Rummukainen et al, 2001), 21\% (Persons et al, 1997), and 86\% (Visscher et al, 1997). The main advantages of gene-specific FISH are high sensitivity and specificity, as well as the possibility to analyze archival formalin-fixed tumor samples. On the other hand, microscopy of FISH is laborious and demanding, and requires an expensive fluorescence microscope. Since the introduction of a novel anticancer drug trastuzumab (Herceptin), analysis of the HER-2/neu oncogene amplification by FISH has become an integral part of breast cancer diagnostics (Tubbs et al, 2001). Because FISH has its technical disadvantages, a new modification of FISH, chromogenic in situ hybridization $(\mathrm{CISH})$, has been developed (Tanner et al, 2000). In CISH the probe detection is based on the use of a peroxidase reaction, the end product of which is visible in conventional light microscopy. FISH and $\mathrm{CISH}$ use similar tissue section pretreatments and probe hybridization protocols and differ only in probe detection. Compared with FISH, CISH offers simple and straightforward microscopic evaluation, potential for concomitant morphologic examination of the target tissue, and simple identification of the cell types showing gene amplification. At present few data are available to verify the accuracy of oncogene amplification detection by $\mathrm{CISH}$ in comparison with FISH; therefore, unlike FISH, $\mathrm{CISH}$ has not yet been widely recommended for 
routine use in clinical decision-making. Here we compared the clinical value of $\mathrm{CISH}$ to $\mathrm{FISH}$ in oncogene amplification screening in tissue arrays using formalinfixed tumor samples as the starting material.

\section{Results}

Altogether 345 primary breast cancer samples placed on four tissue array blocks were analyzed. Due to nonrepresentative histology, detached samples, and unsuccessful hybridizations, 207 (60\%) samples were interpretable by CISH. FISH, performed on adjacent tissue array sections (Rummukainen et al, 2001), was available for 177 of these tumors. Representative illustrations of $\mathrm{CISH}$ and $\mathrm{FISH}$ are shown in Figure 1. c-myc oncogene was found to be amplified in 14.1\% of tumors by $\mathrm{CISH}, 14.0 \%$ by two-color $\mathrm{FISH}$, and in $10.8 \%$ by single-color FISH. Amplification detected by $\mathrm{CISH}$ was highly concordant with both two- and single-color FISH. The kappa coefficients measuring agreement between the assays were 0.76 and 0.67 , respectively (Table 1).

Correlations of c-myc amplification as detected by $\mathrm{CISH}$ or two-color FISH and nine clinicopathologic variables are shown in Table 2. c-myc amplification detected by both assays was significantly associated with high histologic grade, a negative progesterone receptor status, DNA aneuploidy, and a high S-phase fraction. c-myc FISH was associated also with a positive lymph node status, but the association between c-myc amplification detected by $\mathrm{CISH}$ and the lymph node status did not reach statistical significance $(p=0.11)$. The associations between $c-m y c$ amplification as detected by single-color FISH and the

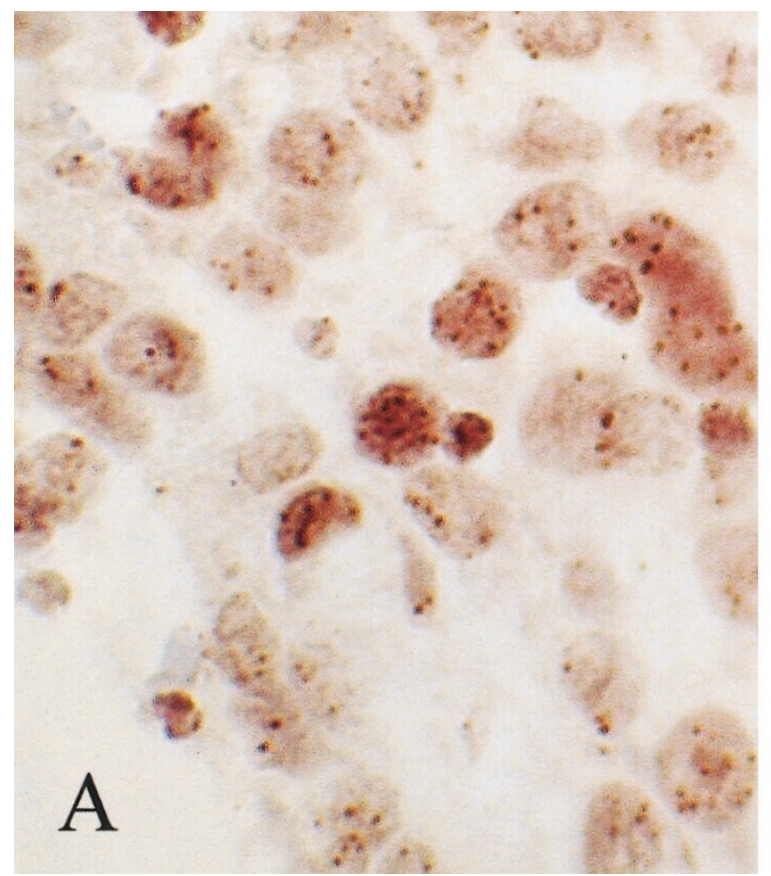

Table 1. Concordance between c-myc Amplification as Assessed by CISH and FISH in 177 Breast Cancers

\begin{tabular}{ccc}
\hline & \multicolumn{2}{c}{ CISH } \\
\cline { 2 - 3 } & No amplification & Amplification \\
\hline Single-color FISH & & \\
No amplification & 151 & 8 \\
Amplification & 1 & 17 \\
Two-color FISH & & 7 \\
No amplification & 145 & 18 \\
Amplification & 7 & \\
\hline
\end{tabular}

FISH, fluorescence in situ hybridization; CISH, chromogenic in situ hybridization.

Kappa coefficient for agreement is 0.76 for single-color FISH and 0.67 for two-color FISH.

clinicopathologic factors were essentially the same (data not shown).

Amplification of c-myc as detected by $\mathrm{CISH}$ was associated with poor distant metastasis-free survival in a univariate survival analysis $(p=0.0013)$, but this association was weaker when amplification was detected by FISH ( $p=0.16$ for two-color FISH and 0.065 for single-color FISH; Fig. 2). In addition, it is noteworthy that a nonamplified but aneuploid copy number of c-myc by $\mathrm{CISH}$ (4-5 copies/cell) was associated with poorer outcome than presence of 1 to 3 copies per cell (Fig. 2C), whereas, when the c-myc copy number was determined by single-color FISH, patients with lowdegree aneuploidy had outcomes similar to patients with no c-myc amplification (Fig. 2B). When c-myc was determined by two-color FISH, patients with

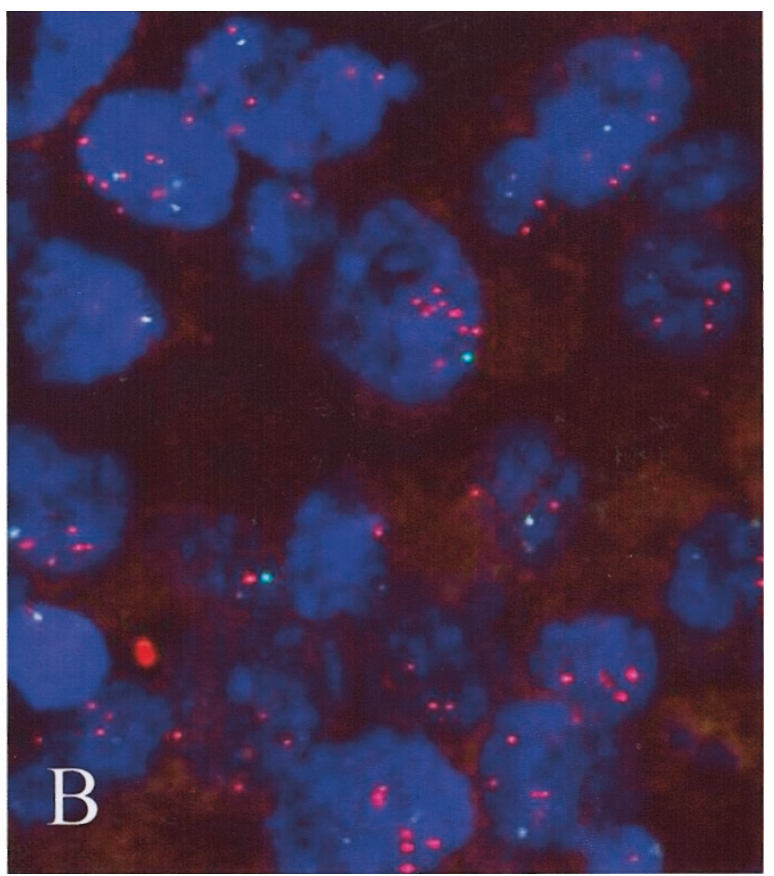

Figure 1.

A tissue array tumor sample showing amplification of c-myc by $\mathrm{CISH}(\mathrm{A})$ and FISH (B). The copy numbers of c-myc range from four to eight copies per cell by both methods. Two-color FISH detected one to two copies of chromosome 8 centromere. Original magnification, $\times 630$. 
Table 2. Association of c-myc Amplification Detected by Two-Color FISH or CISH with Clinicopathological Features in 177 Primary Breast Cancers

\begin{tabular}{|c|c|c|c|c|}
\hline \multirow[b]{2}{*}{ Variable } & \multicolumn{2}{|c|}{ FISH } & \multicolumn{2}{|c|}{$\mathrm{CISH}$} \\
\hline & Amplified ${ }^{a} /$ total & $\begin{array}{l}\text { Odds ratio } \\
\left(p \text { value }^{c}\right)\end{array}$ & Amplified $^{b} /$ total & $\begin{array}{l}\text { Odds ratio } \\
\left(p \text { value }^{c}\right)\end{array}$ \\
\hline All tumors & $27 / 177$ & - & $27 / 177$ & - \\
\hline \multicolumn{5}{|l|}{ Age } \\
\hline$<50$ & $4 / 33$ & & $6 / 33$ & \\
\hline$>50$ & $23 / 144$ & $1.4(0.79)$ & $21 / 144$ & $0.77(0.60)$ \\
\hline \multicolumn{5}{|l|}{ Tumor size } \\
\hline$<2 \mathrm{~cm}$ & $12 / 97$ & & $14 / 97$ & \\
\hline$>2 \mathrm{~cm}$ & $14 / 65$ & $1.9(0.13)$ & $12 / 65$ & $1.3(0.52)$ \\
\hline \multicolumn{5}{|l|}{ Node status } \\
\hline Negative & 9/94 & & $11 / 94$ & \\
\hline Positive & $13 / 60$ & $2.6(0.0057)$ & $13 / 60$ & $2.1(0.11)$ \\
\hline \multicolumn{5}{|c|}{ Distant metastases } \\
\hline No & $25 / 162$ & & $26 / 162$ & \\
\hline Yes & $2 / 5$ & $3.7(0.18)$ & $1 / 5$ & $1.3(0.59)$ \\
\hline \multicolumn{5}{|l|}{ Histologic grade } \\
\hline 1 & $5 / 48$ & & $3 / 48$ & \\
\hline II & $9 / 66$ & $\left(0.0079^{d}\right)$ & $13 / 66$ & $\left(0.026^{d}\right)$ \\
\hline III & $10 / 27$ & & $8 / 27$ & \\
\hline \multicolumn{5}{|c|}{ Estrogen receptor } \\
\hline Negative & $8 / 42$ & & $9 / 42$ & \\
\hline Positive & $14 / 100$ & $0.69(0.45)$ & $14 / 100$ & $0.60(0.32)$ \\
\hline \multicolumn{5}{|c|}{ Progesterone receptor } \\
\hline Negative & $17 / 70$ & & $16 / 70$ & \\
\hline Positive & $5 / 70$ & $0.24(0.0094)$ & $6 / 70$ & $0.32(0.035)$ \\
\hline \multicolumn{5}{|l|}{ DNA ploidy } \\
\hline Diploid & $4 / 57$ & & $2 / 57$ & \\
\hline & $17 / 68$ & $4.4(0.0082)$ & $20 / 68$ & $11.5(0.0001)$ \\
\hline \multicolumn{5}{|l|}{ S-phase fraction } \\
\hline$<12 \%$ & $7 / 72$ & & $5 / 72$ & \\
\hline$>12 \%$ & $13 / 40$ & $4.5(0.0041)$ & $14 / 40$ & $7.2(0.0003)$ \\
\hline
\end{tabular}

FISH, fluorescence in situ hybridization; $\mathrm{CISH}$, chromogenic in situ hybridization.

${ }^{a}$ Samples with two or more extra copies of c-myc compared with the centromere count.

${ }^{b}$ Samples with six or more extra copies of c-myc.

${ }^{c}$ Fisher's exact test.

${ }^{d} \chi^{2}$ test for trend.

low-degree aneuploidy had prognoses similar to patients with a high c-myc copy number (Fig. 2A).

\section{Discussion}

The present study was designed to evaluate the performance of $\mathrm{ClSH}$ in the analysis of c-myc oncogene amplification in a series of archival formalin-fixed breast cancer samples as the starting material. Instead of testing for HER-2/neu oncogene, which is the one most frequently assessed for gene amplification in the clinic, we chose c-myc as the oncogene to be tested, because the c-myc copy numbers are usually lower in tumors showing amplification (6-10 copies/ cell), and such tumors are the most difficult ones to classify correctly by in situ hybridization. Our results showed that $\mathrm{CISH}$ has a high concordance with single- and two-color FISH. The clinicopathologic correlations were essentially the same, and the survival analysis yielded an even stronger association for c-myc amplification determined by $\mathrm{CISH}$ than when assessed by FISH. The overall result was the same when $\mathrm{CISH}$ was compared with either single-color or two-color FISH, the latter including also the differentially labeled reference probe, which is not used in CISH.

Since the early days of gene-specific FISH (Kallioniemi et al, 1992), the use of a reference probe (usually the centromere probe of the respective chromosome) has been considered very important in distinguishing gene amplification from DNA aneuploidy. However, our present results and the recent results by Tubbs et al (2001) clearly indicate that inclusion of the centromere copy number counts affects the gene amplification classification in only a very small fraction of tumors. We have empirically defined six copies per cell as the most suitable cut-off number for the presence of oncogene amplification in CISH (Tanner et al, 2000). In cytogenetic studies of breast cancer (re- 


\section{A. Two-Color FISH}

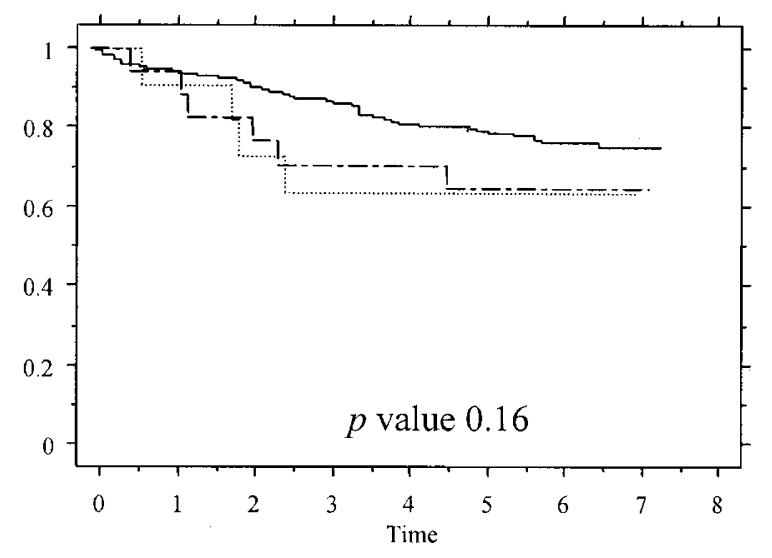

\section{B. One-Color FISH}

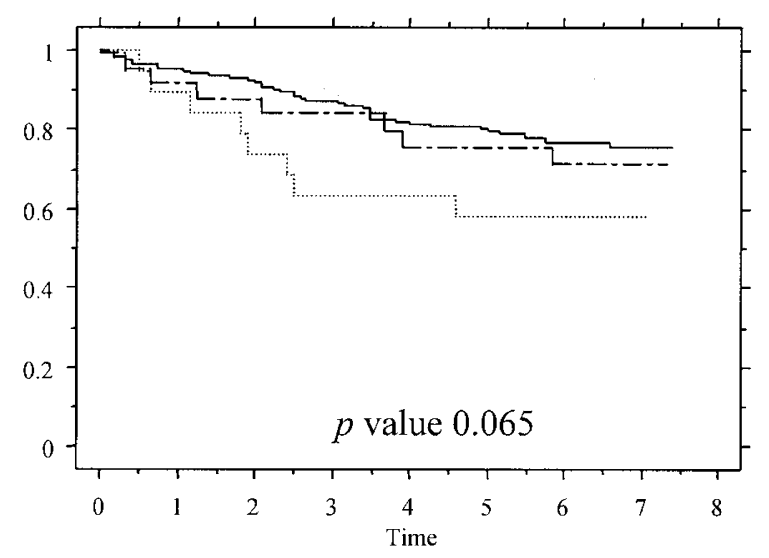

\section{C. $\mathrm{CISH}$}

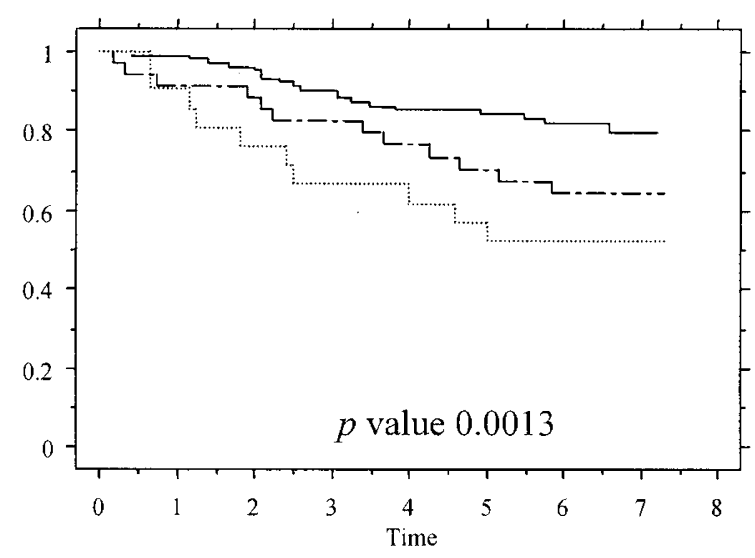

Figure 2.

Kaplan-Maier survival curves of 177 breast cancer patients stratified by the c-myc oncogene copy number. A, Stratification according to two-color FISH. Solid line: no extra copies of c-myc compared with chromosome 8 centromere; broken line: two to four extra copies; and dashed line: five or more extra copies of c-myc. B, Stratification according to single-color FISH. Solid line: one to three copies of c-myc per cell; broken line: an aneuploid copy number of four to five copies per cell; and dashed line: c-myc amplification (six or more copies per cell). C, Stratification according to CISH. c-myc copy numbers classified and illustrated as in B. viewed by Mertens et al, 1997), six-fold polysomies have not been found, which suggests that copy numbers exceeding six cannot be due to DNA aneuploidy. On the other hand, adoption of this cut-off copy number could ignore amplifications of four to five oncogene copies per cell when one to three copies of the reference probe are present. We found 15 such tumors by two-color FISH and, although their survival curve resembled that of the highly c-myc amplified tumors (Fig. 2A), the c-myc amplification by $\mathrm{CISH}$ yielded a stronger association with survival. We anticipate that the better survival stratification by $\mathrm{CISH}$ is attributed to the high sensitivity of the probe detection, excellent visibility of the gene copy signals, and, in particular, the possibility to restrict the copy number counting only on cells that are truly malignant by morphologic criteria (using hematoxylin counterstain). In FISH the malignant nature of the cells included in the copy number analysis is difficult or impossible to verify by the DAPI counterstaining. Therefore, the average oncogene copy numbers detected by FISH may be diluted by the copy numbers of nonmalignant cells, which, in turn, would decrease the average copy number counts.

Although the potential advantages of chromogenic detection over fluorescence detection in DNA ISH have been recognized for many years, the low sensitivity of the chromogenic detection has restricted its use with centromere probes (Hopman et al, 1997). Centromere probes target tens or hundreds of repetitive alpha-satellite sequences, thereby decreasing the sensitivity requirements of the detection system. In gene-specific $\mathrm{CISH}$, which targets a unique DNA sequence, a much higher sensitivity is required for the detection system. We have previously used a genetically modified HER-2/neu oncogene probe, the nonspecific repetitive DNA sequences of which had been depleted (Davison et al, 1998; Tanner et al, 2000). The modification of the probe improves the signal to noise ratio of hybridization several-fold, allowing the use of a conventional three-step detection method (antidigoxigenin-FITC + anti-FITC-HRP + diaminobenzidine [DAB] chromogen). However, when using conventional digoxigenin- or biotin-labeled P1, PAC, and BAC clones, we found this detection method too insensitive and replaced it with a more sensitive one (mouse anti-digoxigenin + anti-mouse HRP polymer + DAB chromogen). The key reagent for higher sensitivity is the anti-mouse-HRP polymer (Powervision), which has a high peroxidase enzyme content as compared with the small size of the polymer molecule. The HRP-polymer has recently been shown to be extremely sensitive in antibody immunohistochemistry (Shi et al, 1999). With the HRP-polymer-based detection, we estimate that the sensitivity of the probe detection in $\mathrm{CISH}$ is close to that of FISH, in which no special signal enhancements are usually needed. This is mainly due to the modern epifluorescence microscopes, with which one can detect minute amounts of label molecules (fluorophores) compared with what is possible in transmitted light microscopy. 
The present results indicate that $\mathrm{CISH}$ is as accurate as FISH in the detection of c-myc oncogene amplification in archival paraffin-embedded breast tumor samples. Because most pathologists are not familiar with fluorescence microscopy but are experienced in evaluating $\mathrm{ClSH}$-like peroxidase-based immunostaining, the time and effort needed for learning to evaluate $\mathrm{CISH}$ is much shorter than for FISH. The practical advantages of $\mathrm{CISH}$ became evident also during the current study when we needed to analyze hundreds of tissue array samples both by conventional light microscopy for $\mathrm{CISH}$ and by the fluorescence microscopy for FISH. Microscopy for $\mathrm{CISH}$ turned out to be relatively fast and straightforward, whereas the fluorescence microscopy of tissue arrays, which otherwise provide an excellent tool to evaluate hybridization methods, was relatively tedious and cumbersome. This was mainly due to the inability to use fluorescence microscope with low magnification objectives, which are needed for finding the most representative sample areas and for moving the focus from one tissue array spot to another.

In conclusion, the present study shows that detection of c-myc amplification by CISH leads to meaningful clinical correlations and that breast cancers found to have c-myc amplification by CISH may be associated with poorer outcome as compared with the amplification status determined by either single-color or two-color FISH. CISH analysis is technically easier and less time consuming than FISH and allows evaluation of the tissue architecture with an ordinary transmitted light microscope. Although further comparative studies on other clinically important oncogenes such as the HER-2/neu are needed, the present results suggest that $\mathrm{CISH}$ may eventually replace FISH in oncogene screening studies on large tumor materials, and tissue arrays and, ultimately, in clinical diagnostics as a more simple and economic technique that allows direct identification of the cells showing gene amplification.

\section{Materials and Methods}

\section{Patients and Tumors}

Archival formalin-fixed paraffin-embedded tumor blocks from 345 primary breast cancer patients were collected from the pathology laboratories of the Tampere University Hospital district. According to the data files of the Finnish Cancer Registry, these tumors represent $84.2 \%$ of all primary invasive breast cancers operated in the Tampere University Hospital district in 1991 and 1992 (population approximately 400,000). The mean age of patients was 61.6 years. The histologic type was available for 241 tumors including 198 (82\%) ductal, 21 (9\%) lobular, and 22 (9\%) tumors of the special histologic types. The collected clinicopathologic data (Lundin et al, 2001) included patient age at the time of diagnosis, postoperative tumor size, axillary nodal status and presence of distant metastases at the time of the diagnosis (PTNM), histologic grade, estrogen (ER) and progesterone (PR) receptor status,
DNA ploidy, and the S-phase fraction (SPF) size. The median follow-up time for the patients still alive was 6.8 years (range, $5.1-7.8$ years).

\section{Preparation of Tissue Arrays}

Multitissue array blocks were produced as previously described (Rummukainen et al, 2001). In short, representative tumor regions were selected from hematoxylin and eosin-stained sections for implantation in the multitumor array blocks. One tissue cylinder with a diameter of $0.6 \mathrm{~mm}$ was punched through the selected tumor area from each donor tissue block. One tissue core from each donor block was then inserted into four recipient tissue array paraffin blocks, resulting in a total of 50 to 144 tumor samples into each recipient block (Kononen et al, 1998). Sections of the recipient block were cut at $5 \mu \mathrm{m}$, collected on SuperFrost Plus glass slides, and baked in a $60^{\circ} \mathrm{C}$ oven for 2 to 4 hours before starting the FISH and $\mathrm{CISH}$ procedures.

\section{$\mathrm{CISH}$}

The protocol used has been modified and improved from Tanner et al (2000). Before hybridization, the tissue sections were deparaffinized in xylene, washed in $100 \%$ ethanol, and air-dried. Slides were then incubated in $0.1 \mathrm{M}$ Tris-saline $(\mathrm{pH} 7.3)$ at $121^{\circ} \mathrm{C}$ in an autoclave for 2 minutes. After cooling at room temperature for 15 minutes and rinsing twice in PBS for 3 minutes, the tissue sections were covered with $100 \mu \mathrm{l}$ of pepsin solution (Digest-All 3; Zymed, South San Francisco, California) at $37^{\circ} \mathrm{C}$ for 6 to 8 minutes. The slides were then washed in PBS three times at room temperature for 2 minutes, dehydrated in graded ethanols, and air dried. Ten microliters of probe cocktail $(2 \mu \mathrm{l}$ of digoxigenin-labeled $\mathrm{P} 1$ probe to c-myc oncogene RMC08p001, $1 \mu \mathrm{l}$ of $9.9 \mu \mathrm{g} / \mu \mathrm{l}$ of human placental DNA, and $1 \mu \mathrm{l}$ of $1 \mu \mathrm{g} / \mu \mathrm{l}$ of Cot-1 DNA [Roche Molecular Biochemicals, Mannheim, Germany], and $6 \mu \mathrm{l}$ of master mix [Rummukainen et al, 2001]) was applied to the tissue sections. Slides were coverslipped and sealed with rubber cement to prevent evaporation of the probe solution. Slides and the probe mixture were codenaturated at $94^{\circ} \mathrm{C}$ for 3 minutes on a thermal plate, and hybridization was carried out in a humid chamber at $37^{\circ} \mathrm{C}$ for 36 to 40 hours (over two nights). After hybridization, the slides were washed with $0.5 \times \mathrm{SSC}$ for 5 minutes in $75^{\circ} \mathrm{C}$, followed by three washes in PBS for 2 minutes at room temperature. c-myc probe was detected with sequential incubations with mouse anti-digoxigenin (Roche Biochemicals; diluted 1:300 in PowerVision Blocking solution), goat anti-mouse HRP polymer (Powervision; ImmunoVision Technologies, Daly City, California), and DAB (ImmunoVision Technologies) as chromogens. Incubation in DAB Enhancer (Zymed) for 3 minutes was used to further enhance the signal intensity. After light counterstaining with hematoxylin, the slides were dehydrated and embedded. 
$\mathrm{CISH}$ hybridizations were analyzed using a Nicon Labophot transmitted light microscope with a $40 \times$ objective. At least 50 nonoverlapping nuclei in every tumor sample were scored to determine the number of c-myc signals. The results were expressed as the actual copy numbers per cell in each sample. Amplification was defined to be present when six or more copies of c-myc were detected in at least $20 \%$ of the screened malignant cells.

\section{FISH}

Two-color FISH was performed using the digoxigeninlabeled P1 probe to c-myc gene (RMC08p001) and a FITC-labeled probe to chromosome 8 centromere (pJM128; ATCC, Rockville, Maryland) (Rummukainen et al, 2001). To improve the success rate of FISH, a commercially available c-myc probe (LSI c-myc; Vysis, Downers Grove, Illinois) and the FITC-labeled pJM128 for chromosome 8 centromere were used on a separate set of tissue sections, as previously described (Rummukainen et al, 2001). Copy number counts with these two probes were virtually identical, and the FISH result was recorded if either probe set yielded a satisfactory hybridization result. The microscopic analysis was performed using an Olympus BX50 epifluorescence microscope (with $\times 60$ oil immersion objective), equipped with a CCD camera (Photometrics, Tucson, Arizona). The slides were analyzed using two different methods: two-color FISH (c-myc and centromere 8) and single-color FISH (c-myc only). In twocolor FISH, the average c-myc copy number was compared with the centromere 8 copy number count. Amplification was defined to be present when at least two extra copies of c-myc were seen in at least $20 \%$ of the tumor cells. In single-color FISH the presence of six or more copies of c-myc was considered as amplification. All c-myc analyses were carried out blinded to the clinical outcome of the patients.

\section{Statistics}

Contingency tables were analyzed with Fisher's exact test. The $\chi^{2}$ test for trend was used to compare the frequency of c-myc amplification with histologic grade. The analysis of distant disease-free survival was performed using Kaplan-Maier survival analysis, and survival of different groups was compared with the log-rank test. All $p$ values are two-tailed.

\section{Acknowledgement}

The skillful technical assistance of Mrs. Anne Luuri, Mrs. Sari Toivola, and Ms. Päivi Järvinen is greatly appreciated.

\section{References}

Davison JM, Morgan TW, Hsi BL, Xiao S, and Fletcher JA (1998). Subtracted, unique-sequence, in situ hybridization: Experimental and diagnostic applications. Am J Pathol 153: 1401-1409.
Deming SL, Nass SJ, Dickson RB, and Trock BJ (2000). C-myc amplification in breast cancer: A meta-analysis of its occurrence and prognostic relevance. $\mathrm{Br} \mathrm{J}$ Cancer 83:16881695.

Escot C, Theillet C, Lidereau R, Spyratos F, Champeme MH, Gest J, and Callahan R (1986). Genetic alteration of the c-myc proto-oncogene (MYC) in human primary breast carcinomas. Proc Natl Acad Sci USA 83:4834-4838.

Hopman AH, Claessen S, and Speel EJ (1997). Multi-colour brightfield in situ hybridisation on tissue sections. Histochem Cell Biol 108:291-298.

Kallioniemi OP, Kallioniemi A, Kurisu W, Thor A, Chen LC, Smith HS, Waldman FM, Pinkel D, and Gray JW (1992). ERBB2 amplification in breast cancer analyzed by fluorescence in situ hybridization. Proc Natl Acad Sci USA 89:53215325.

Kononen J, Bubendorf L, Kallioniemi A, Barlund M, Schraml P, Leighton S, Torhorst J, Mihatsch MJ, Sauter G, and Kallioniemi OP (1998). Tissue microarrays for highthroughput molecular profiling of tumor specimens. Nat Med 4:844-847.

Lundin J, Lundin M, Holli K, Kataja V, Elomaa L, Pylkkanen L, Turpeenniemi-Hujanen T, and Joensuu H (2001). Omission of histologic grading from clinical decision making may result in overuse of adjuvant therapies in breast cancer: Results from a nationwide study. J Clin Oncol 19:28-36.

Mertens F, Johansson B, Hoglund M, and Mitelman F (1997). Chromosomal imbalance maps of malignant solid tumors: $A$ cytogenetic survey of 3185 neoplasms. Cancer Res 57:27652780.

Nesbit CE, Tersak JM, and Prochownik EV (1999). MYC oncogenes and human neoplastic disease. Oncogene 18: 3004-3016.

Persons DL, Borelli KA, and Hsu PH (1997). Quantitation of HER-2/neu and c-myc gene amplification in breast carcinoma using fluorescence in situ hybridization. Mod Pathol 10:720-727.

Press MF, Bernstein L, Thomas PA, Meisner LF, Zhou JY, Ma Y, Hung G, Robinson RA, Harris C, El-Naggar A, Slamon DJ, Phillips RN, Ross JS, Wolman SR, and Flom KJ (1997). HER-2/neu gene amplification characterized by fluorescense in situ hybridization: Poor prognosis in node-negative breast carcinomas. J Clin Oncol 15:2894-2904.

Rummukainen J, Salminen T, Lundin J, Kytölä S, Joensuu H, and Isola J (2001). Amplification of c-myc by fluorescence in situ hybridization in a population-based breast cancer tissue array. Mod Pathol 14:1030-1035.

Schraml P, Kononen J, Bubendorf L, Moch H, Bissig $\mathrm{H}$, Nocito A, Mihatsch MJ, Kallioniemi OP, and Sauter G (1999). Tissue microarrays for gene amplification surveys in many different tumor types. Clin Cancer Res 5:1966-1975.

Shi SR, Guo J, Cote RJ, Young LL, Hawes D, Shi Y, Thu S, and Taylor CR (1999). Sensitivity and detection efficiency of a novel two-step detection system (Powervision) for immunohistochemistry. Appl Immunohistochem Molecul Morphol 7:201-208.

Tanner M, Gancberg D, Di Leo A, Larsimont D, Rouas G, Piccart MJ, and Isola J (2000). Chromogenic in situ hybridization: A practical alternative for fluorescence in situ hybridization to detect HER-2/neu oncogene amplification in archival breast cancer samples. Am J Pathol 157:1467-1472. 
Tubbs RR, Pettay JD, Roche PC, Stoler MH, Jenkins RB, and Grogan TM (2001). Discrepancies in clinical laboratory testing of eligibility for trastuzumab therapy: Apparent immunohistochemical false-positives do not get the message. J Clin Oncol 19:2714-2721.
Visscher DW, Wallis T, Awussah S, Mohamed A, and Crissman JD (1997). Evaluation of MYC and chromosome 8 copy number in breast carcinoma by interphase cytogenetics. Genes Chromosomes Cancer 18:1-7. 